\title{
ON THE IMBEDDING OF A DIRECT PRODUCT INTO A ZERO-DIMENSIONAL COMMUTATIVE RING
}

\author{
ROBERT GILMER AND WILLIAM HEINZER
}

(Communicated by Louis J. Ratliff, Jr.)

\begin{abstract}
This paper addresses questions related to results of $M$. Arapovic concerning imbeddability of a commutative unitary ring $R$ in a zero-dimensional ring. The case where $R$ is a product of zero-dimensional rings is of special interest. We show (1) if the zero ideal of $R$ admits a unique representation as an irredundant intersection of (strongly primary) ideals, then $R$ need not be imbeddable in a zero-dimensional ring, and (2) for a family $\left\{R_{\alpha}\right\}$ of zerodimensional rings, $R=\prod R_{\alpha}$ is imbeddable in a zero-dimensional ring if and only if $R$ itself is zero-dimensional.
\end{abstract}

All rings considered in this paper are assumed to be commutative and unitary. In his recently published book [6, §3], J. Huckaba considers the problems of determining those rings $R$ that can be imbedded in a zero-dimensional ring $T$, where it is understood that the imbedding is unitary in the sense that it maps the identity element of $R$ to the identity element of $T$. The main result obtained, Theorem 3.5, is due to Arapovic [3, Theorem 7]; it states that $R$ is so imbeddable if and only if there exists a family $\left\{Q_{\lambda}\right\}$ of ideals of $R$, where $Q_{\lambda}$ is $P_{\lambda}$-primary, such that the following conditions (A1) and (A2) are satisfied:

(A1) $\cap_{\lambda} Q_{\lambda}=(0)$.

(A2) For each element $a \in R$, there exists a positive integer $n_{a}$ such that $a^{n_{a}} \notin \bigcup_{\lambda}\left(P_{\lambda} \backslash Q_{\lambda}\right)$.

We note that condition (A1) implies that $R$ is imbeddable in $\prod_{\lambda}\left(R / Q_{\lambda}\right)_{P_{\lambda} / Q_{\lambda}}$, the product of a family of zero-dimensional rings; such a product need not be zero-dimensional, and in fact, Maroscia in [7, Proposition 2.6] shows that a product $S=\prod S_{\alpha}$ of zero-dimensional rings $S_{\alpha}$ is zero-dimensional if and only if $N(S)=\prod N\left(S_{\alpha}\right)$, where $N(\cdot)$ denotes the nilradical. (In [4], we show that if $\operatorname{dim} S \neq 0$, then $\operatorname{dim} S=\infty$.) Because of this imbedding property of $R$, however, and because condition (A2) represents a kind of uniform bound on the index of nilpotency of elements of $P_{\lambda}$ modulo $Q_{\lambda}$, we were led to ask

Received by the editors June 24, 1988 and, in revised form, November 29, 1988.

1980 Mathematics Subject Classification (1985 Revision). Primary 13B02; Secondary 13A99, $13 \mathrm{~B} 99$.

Key words and phrases. Imbedding, zero-dimensional rings, products of commutative rings.

The research of the first author was partially supported by NSF Grant DMS-8501003.

The research of the second author was partially supported by NSF Grant DMS-8521767. 
whether a ring $T$ can be imbedded in a zero-dimensional ring if the zero ideal of $T$ is an intersection of strongly primary ideals. (Recall that an ideal $Q$ of $T$ is strongly primary if $Q$ is primary and contains a power of its radical.) We show in Example 4 that this question has a negative answer. It also seemed natural to us to ask for conditions under which $\Pi S_{\alpha}$ is imbeddable in a zerodimensional ring, particularly in the case where each $S_{\alpha}$ is zero-dimensional. Theorems 3,7 and 10 , as well as Corollary 8 , provide such conditions, and in particular, Corollary 8 shows that if each $S_{\alpha}$ is zero-dimensional, then $\Pi S_{\alpha}$ is imbeddable in a zero-dimensional ring if and only if $\Pi S_{\alpha}$ itself is zero-dimensional.

Our first result shows that restriction to unitary imbeddings into a zerodimensional ring is, in fact, not a substantial restriction.

Proposition 1. If there exists an isomorphism of the ring $R$ into a zero-dimensional ring, then there exists a unitary imbedding of $R$ into a zero-dimensional ring.

Proof. Let $\alpha$ be an isomorphism of $R$ into the zero-dimensional ring $S$. If $e$ is the identity element of $R$, then $\alpha(e)$ is an idempotent of $S$ and $\alpha(R) \subseteq \alpha(e) S$, a subring of $S$ with identity element $\alpha(e)$. Moreover, since $S=\alpha(e) S \oplus(1-\alpha(e)) S$, the ring $\alpha(e) S$ is zero-dimensional. Therefore $\alpha$ is a unitary imbedding of $R$ into the zero-dimensional ring $\alpha(e) S$.

We will use the following consequence of Proposition 1: if $R$ is a subring of the ring $S$, not necessarily with the same identity element that $S$ has, and if $R$ is not imbeddable in a zero-dimensional ring, then neither is $S$.

If $R=\prod_{\alpha \in A} R_{\alpha}$ and if $\alpha \in A$, we use $e_{\alpha}$ to denote the idempotent of $R$ with 1 in the $\alpha$ th coordinate and zeroes elsewhere. If $r \in R_{\alpha}, r * e_{\alpha}$ denotes the element of $R$ with $r$ in the $\alpha$ th coordinate and 0 in all other coordinates, and for $I \subseteq R_{\alpha}, I * e_{\alpha}$ is defined to be $\left\{i * e_{\alpha} \mid i \in I\right\}$. The next proposition, an auxiliary result used in the proof of Theorem 3 , uses this notation. We omit the routine proof of Proposition 2.

Proposition 2. Suppose $\left\{Q_{\lambda}\right\}_{\lambda \in \Lambda}$ is a family of primary ideals of $R=\prod_{\alpha \in A} R_{\alpha}$ satisfying condition $(A 1)$.

(1) For each $\alpha \in A$, the set $\Lambda_{\alpha}=\left\{\lambda \in \Lambda \mid 1-e_{\alpha} \in Q_{\lambda}\right\}$ is nonempty.

(2) For $\lambda \in \Lambda_{\alpha}$, the ideal $Q_{\lambda}$ is of the form $\left(H_{\lambda} * e_{\alpha}\right)+\left(1-e_{\alpha}\right) R$, where $H_{\lambda}$ is a primary ideal of $R_{\alpha}$.

(3) The family $\left\{H_{\lambda}\right\}_{\lambda \in \Lambda_{r}}$ satisfies (A1), and if $R$ satisfies (A2) with respect to $\left\{Q_{\lambda}\right\}_{\lambda \in \Lambda}$, then $R_{\alpha}$ satisfies (A2) with respect to $\left\{H_{\lambda}\right\}_{\lambda \in \Lambda_{\alpha}}$.

(4) $\bigcap_{\lambda \in \Lambda_{t}} Q_{\lambda}=\left(1-e_{\alpha}\right) R$. Hence if $\Sigma=\bigcap_{\alpha \in A} \Lambda_{\alpha}$, then $\left\{Q_{\lambda}\right\}_{\lambda \in \Sigma}$ satisfies (A1). Consequently, the ideals $Q_{\lambda}$, for $\lambda \in \Lambda \backslash \Sigma$, are redundant in the representation $(0)=\bigcap_{\lambda \in \Lambda} Q_{\lambda}$.

(5) If $R$ does not satisfy $(A 2)$ with respect to $\left\{Q_{\lambda}\right\}_{\lambda \in \Sigma}$, then neither does $R$ satisfy $(A 2)$ with respect to $\left\{Q_{\lambda}\right\}_{\lambda \in \Lambda}$. 
The statement of Theorem 3 employs the following terminology and notation. A ring $R$ is primary [10, p. 204] if $R$ contains a unique proper prime ideal; equivalently, $R$ is primary if $R$ is zero-dimensional and quasi-local. If $x \in N(R)$, we denote by $\eta(x)$ the index of nilpotency of $x$-that is, $\eta(x)=k$ if $x^{k}=0$ but $x^{k-1} \neq 0$. We define $\eta(R)$ to be $\sup \{\eta(x) \mid x \in N(R)\}$; if the set $\{\eta(x) \mid x \in N(R)\}$ is unbounded, then we write $\eta(R)=\infty$.

Theorem 3. Let $\left\{\left(R_{\alpha}, P_{\alpha}\right)\right\}_{\alpha \in A}$ be a family of primary rings and let $R=\prod R_{\alpha}$. The following conditions are equivalent.

(1) $R$ is imbeddable in a zero-dimensional ring.

(2) There exists a positive integer $n$ such that $\left\{\alpha \in A \mid \eta\left(R_{\alpha}\right)>n\right\}$ is finite.

Proof. We let $\eta\left(R_{\alpha}\right)=k_{\alpha}$. Suppose (2) is satisfied, let $B=\left\{\alpha \in A \mid k_{\alpha}>n\right\}$ $=\left\{\alpha_{1}, \ldots, \alpha_{t}\right\}$, and let $C=A \backslash B$. Then $R \simeq R_{\alpha_{1}} \oplus \cdots \oplus R_{\alpha_{t}} \oplus S$, where $S=\prod_{\alpha \in C} R_{\alpha}$. We note that if $x \in \prod_{\alpha \in C} N\left(R_{\alpha}\right)$, then $x^{n}=0$ by definition of $C$, and hence $x \in N(S)$. Since the inclusion $N(S) \subseteq \prod N\left(R_{\alpha}\right)$ always holds, we conclude that $N(S)=\prod_{\alpha \in C} N\left(R_{\alpha}\right)$, and by the result of Maroscia [7] cited previously, $\operatorname{dim} S=0$. Therefore $\operatorname{dim} R=0$ as well, and (1) is satisfied.

To prove that (1) implies (2), we establish the contrapositive. Thus, assume that $\left\{\alpha \in A \mid k_{\alpha}>n\right\}$ is infinite for each positive integer $n$. Then there exist distinct elements $\alpha_{1}, \alpha_{2}, \ldots$ in $A$ such that $k_{\alpha_{i}}>i$ for each $i$. Since $\prod_{i=1}^{\infty} R_{\alpha_{i}}$ is isomorphic to a subring of $R$, it follows from Proposition 1 that it suffices to show that $\prod R_{\alpha_{i}}$ is not imbeddable in a zero-dimensional ring. Writing $i$ in place of $\alpha_{i}$, we therefore assume without loss of generality that $A=\mathbf{Z}^{+}$, the set of positive integers. Since $k_{i}>i$, we can choose an element $y_{i} \in P_{i}$ such that $y_{i}^{i} \neq 0$ for each $i$. Let $y$ be the element $\left\{y_{i}\right\}_{i=1}^{\infty}$ of $R$. To show that $R$ is not imbeddable in a zero-dimensional ring, we show that if $\left\{Q_{\lambda}\right\}_{\lambda \in \Lambda}$ is any family of primary ideals of $R$ satisfying (A1), then condition (A2) is not satisfied with respect to $\left\{Q_{\lambda}\right\}$ for the element $y$. Thus, Proposition 2 shows that for each $i,\left\{Q_{\lambda}\right\}$ contains a family $\left\{\left(H_{\lambda} * e_{i}\right)+\left(1-e_{i}\right) R\right\}_{\lambda \in \Lambda_{i}}$, where $H_{\lambda}$ is a primary ideal of $R_{i}$ and $\cap_{\lambda \in \Lambda_{i}} H_{\lambda}=(0)$. Let $s$ be any positive integer. Then $y^{s}=\left\{y_{i}^{s}\right\}$. Since $\cap_{\lambda \in \Lambda_{s}} H_{\lambda}=(0)$, there exists $\lambda_{0} \in \Lambda_{s}$ such that $y_{s}^{s} \notin H_{\lambda_{0}}$. Consequently, $y_{s}^{s} \in P_{s} \backslash H_{\lambda_{0}}$ and $y^{s} \in\left[\left(P_{s} * e_{s}\right)+\left(1-e_{s}\right) R\right] \backslash\left[\left(H_{\lambda_{0}} * e_{s}\right)+\left(1-e_{s}\right) R\right]$, where $\left(P_{s} * e_{s}\right)+\left(1-e_{s}\right) R$ is the radical of $\left(H_{\lambda_{0}} * e_{s}\right)+\left(1-e_{s}\right) R$. Because $\left(H_{\lambda_{0}} * e_{s}\right)+\left(1-e_{s}\right) R \in\left\{Q_{\lambda}\right\}$, we conclude that $y^{s} \in \cup\left(P_{\lambda} \backslash Q_{\lambda}\right)$ for each $s \in \mathbf{Z}^{+}$. Thus (A2) fails for $y$ and $\left\{Q_{\lambda}\right\}, R$ is not imbeddable in a zero-dimensional ring, and this completes the proof of Theorem 3.

Using Theorem 3, we can give an example of a ring $T$ such that the zero ideal of $T$ admits a unique irredundant representation as an intersection of (strongly) primary ideals, but $T$ is not imbeddable in a zero-dimensional ring. Thus, condition (A1) alone does not imply imbeddability, even if the ideals 
$Q_{\lambda}$ are strongly primary and the intersection representation $(0)=\bigcap_{\lambda} Q_{\lambda}$ is irredundant.

Example 4. For $n \in \mathbf{Z}^{+}$, let $T_{n}=\mathbf{Z} / p^{n} \mathbf{Z}$, where $p$ is prime, and let $T=$ $\prod_{1}^{\infty} T_{n}$. Since $\eta\left(T_{n}\right)=n$, Theorem 3 shows that $T$ is not imbeddable in a zerodimensional ring. Moreover, since any family of ideals of $T_{n}$ with intersection (0) must contain the ideal (0) itself, it follows from Proposition 2 that if $\left\{Q_{\lambda}\right\}$ is any family of primary ideals of $T$ satisfying (A1), then $\left\{Q_{\lambda}\right\} \supseteq\left\{\left(1-e_{i}\right) T\right\}_{1}^{\infty}$. We observe that $(0)=\bigcap_{1}^{\infty}\left(1-e_{i}\right) T$ is an irredundant representation of $(0)$ as an intersection of primary ideals; Proposition 2 implies that this representation is unique. Finally, we note that $\left(1-e_{i}\right) T$ contains the $i$ th power of its radical $\left(p T_{i} * e_{i}\right)+\left(1-e_{i}\right) T$, and hence is strongly primary.

The ring $T$ of Example 4 is infinite-dimensional, but by appropriately modifying $T$, we can obtain rings of arbitrary positive dimension that share properties of $T$, as described in Example 4.

Example 5. Let $T$ and $T_{n}$ be as in Example 4, and let $\pi$ denote the prime subring of $T$. Since $\operatorname{char} T=0, \pi \simeq \mathbf{Z}$. If $I$ denotes the direct sum of $\left\{T_{n}\right\}_{n=1}^{\infty}$ within $T$, then $I$ is an ideal of $T$ and elements of $I$ are integral over $\pi$. Let $S=\pi+I$. Then $\operatorname{dim} S=1$, and as in Proposition 2 and Example 4, we can argue that if $\left\{Q_{\lambda}\right\}$ is any family of primary ideals of $S$ with intersection (0), then $\left\{\left(1-e_{i}\right) S\right\}_{i=1}^{\infty} \subseteq\left\{Q_{\lambda}\right\}$. If $e$ denotes the identity element of $S$, then pe does not satisfy condition (A2) with respect to the family $\left\{\left(1-e_{i}\right) S\right\}_{1}^{\infty}$, nor to the family $\left\{Q_{\lambda}\right\}$. Hence $S$ is not imbeddable in a zero-dimensional ring, but as in Example 4, (0) admits the unique irredundant representation $(0)=\bigcap_{1}^{\infty}\left(1-e_{i}\right) S$ as an intersection of (strongly) primary ideals. For $n \in \mathbf{Z}^{+}$, the ring $S_{n}=S\left[X_{1}, \ldots, X_{n}\right]$ is integral over $\pi\left[X_{1}, \ldots, X_{n}\right]$, and hence $\operatorname{dim} S_{n}=n+1$. Because $S$ is not imbeddable in a zero-dimensional ring, neither is $S_{n}$. Moreover, it is again the case that the zero ideal of $S_{n}$ admits the unique irredundant representation $(0)=\bigcap_{1}^{\infty}\left(1-e_{i}\right) S_{n}$ as an intersection of (strongly) primary ideals.

Our next goal is to extend, in Corollary 8, Theorem 3 to the case of a product $\prod R_{\alpha}$ of arbitrary zero-dimensional rings. Theorem 7 provides an avenue to this goal. The proof of Theorem 7 uses Lemma 6. Again because it is straightforward, we omit the proof of Lemma 6.

Lemma 6. Assume that the zero ideal of $R$ is the intersection of a family $\left\{Q_{\lambda}\right\}_{\lambda \in \Lambda}$ of primary ideals of $R$, where $Q_{\lambda}$ is $P_{\lambda}$-primary. Then $\eta(R)=\sup \left\{\eta\left(R_{P_{\lambda}}\right)\right\}_{\lambda \in \Lambda}$.

We remark that if $\prod R_{\alpha}$ is imbeddable in a zero-dimensional ring, then so is each $R_{\alpha}$ (Proposition 1). Hence the hypothesis in Theorems 7 and 10 that each $R_{\alpha}$ is imbeddable in a zero-dimensional ring is necessary for the conclusion that $\prod R_{\alpha}$ is so imbeddable. 
Theorem 7. Let $\left\{R_{\alpha}\right\}_{\alpha \in A}$ be a family of rings, each imbeddable in a zerodimensional ring. For each $\alpha \in A$, assume that the zero ideal of $R_{\alpha}$ is an intersection of primary ideals belonging to height-zero prime ideals. Let $R=\prod R_{\alpha}$. The following conditions are equivalent.

(1) $R$ is imbeddable in a zero-dimensional ring.

(2) There exists a positive integer $n$ such that the set $\left\{\alpha \in A \mid \eta\left(R_{\alpha}\right)>n\right\}$ is finite.

Proof. (1) $\Rightarrow(2)$. We prove the contrapositive. Thus, assume that $\left\{\alpha \in A \mid \eta\left(R_{\alpha}\right)>n\right\}$ is infinite for each $n \in \mathbf{Z}^{+}$. Then there exist distinct elements $\alpha_{1}, \alpha_{2}, \ldots$ of $A$ such that $\eta\left(R_{\alpha_{i}}\right)>i$ for each $i$. As in the proof of Theorem 3, it suffices to show that $\prod_{1}^{\infty} R_{\alpha_{i}}$ is not imbeddable in a zerodimensional ring, and writing $i$ in place of $\alpha_{i}$, we assume without loss of generality that $A=\mathbf{Z}^{+}$. For each $i$, choose $x_{i} \in N\left(R_{i}\right)$ such that $x_{i}^{i} \neq 0$. By hypothesis on $R_{i}$, there exists a primary ideal $Q_{i}$ of $R_{i}$, with radical $P_{i}$ of height zero, such that $x_{i}^{i} \notin Q_{i}$. Let $S_{i}=R_{i} \backslash P_{i}$ for each $i$ and let $S$ be the multiplicative system $\prod_{1}^{\infty} S_{i}$ in $R$. If $R$ were imbeddable in a zero dimensional ring $T$, then $R_{S} \simeq \prod_{1}^{\infty}\left(R_{i}\right)_{P_{i}}$ would be imbeddable in the zerodimensional ring $T_{s}$. However, $\left(R_{i}\right)_{P_{i}}$ is a primary ring and $x_{i} / 1$ is a nilpotent element of this ring such that $\left(x_{i} / 1\right)^{i} \neq 0$, hence $\eta\left(\left(R_{i}\right)_{P_{i}}\right)>i$. By Theorem 3, we conclude that $\prod_{1}^{\infty}\left(R_{i}\right)_{P_{i}}$, ergo $R$, is not imbeddable in a zerodimensional ring.

(2) $\Rightarrow$ (1). Let $N \in \mathbf{Z}^{+}$be such that $B=\left\{\alpha \in A \mid \eta\left(R_{\alpha}\right)>n\right\}=\left\{\alpha_{i}\right\}_{i=1}^{t}$ is finite. If $C=A \backslash B$, then $R \cong R_{\alpha_{1}} \oplus \cdots \oplus R_{\alpha_{t}} \oplus\left(\prod_{\alpha \in C} R_{\alpha}\right)$. Since each $R_{\alpha_{i}}$ is imbeddable in a zero-dimensional ring $T_{i}$, it follows that if $\prod_{\alpha \in C} R_{\alpha}$ is imbeddable in a zero-dimensional ring $T$, then $R$ is imbeddable in the zerodimensional ring $T_{1} \oplus \cdots \oplus T_{t} \oplus T$. Hence, without loss of generality we assume that $A=C$. Then for $\alpha \in A$, there exists a family $\left\{Q_{\lambda}^{(\alpha)}\right\}_{\lambda \in \Lambda_{\kappa}}$ of primary ideals of $R_{\alpha}$ such that $\bigcap_{\lambda} Q_{\lambda}^{(\alpha)}=(0)$, where $P_{\lambda}^{(\alpha)}=\sqrt{Q_{\lambda}^{(\alpha)}}$ has height zero. For each $\lambda \in \Lambda_{\alpha}$, denote by $R_{\lambda}^{(\alpha)}$ the localization of the ring $R_{\alpha} / Q_{\lambda}^{(\alpha)}$ at the prime ideal $P_{\lambda}^{(\alpha)} / Q_{\lambda}^{(\alpha)}$. Then $R_{\lambda}^{(\alpha)}$ is a primary ring, and $R_{\alpha}$ is imbedded in $\prod_{\lambda \in \Lambda_{a}} R_{\lambda}^{(\alpha)}$. Moreover, since $R_{\lambda}^{(\alpha)}$ is a homomorphic image of the primary ring $\left(R_{\alpha}\right)_{P_{i}^{(n)}}$, we have $\eta\left(R_{\lambda}^{(\alpha)}\right) \leq \eta\left(\left(R_{\alpha}\right)_{\left.P_{\lambda}^{(n)}\right)} \leq \eta\left(R_{\alpha}\right) \leq n\right.$, where the penultimate inequality is obtained from Lemma 6. Finally, $R$ is imbedded in $\prod_{\alpha \in A}\left(\prod_{\lambda \in \Lambda_{n}} R_{\lambda}^{(\alpha)}\right)$, and Theorem 3 shows that this latter ring is imbeddable in a zero-dimensional ring. (The proof of Theorem 3 shows, in fact, that the latter ring is zero-dimensional.) This completes the proof.

Corollary 8. If $\left\{R_{\alpha}\right\}_{\alpha \in A}$ is a family of zero-dimensional rings and if $R=\prod R_{\alpha}$, the following conditions are equivalent.

(1) $\operatorname{dim} R=0$.

(2) $R$ is imbeddable in a zero-dimensional ring. 
(3) There exists a positive integer $n$ such that $\left\{\alpha \in A \mid \eta\left(R_{\alpha}\right)>n\right\}$ is finite. Proof. The equivalence of (2) and (3) follows from Theorem 7, and clearly (1) implies (2). As in the proof of Theorem 3, condition (3) implies that $N(R)=$ $\prod N\left(R_{\alpha}\right)$, and hence (1) follows from (3).

The proof of Theorem 7 establishes the next result. The converse of Corollary 9 is valid if each $R_{\alpha}$ is zero-dimensional.

Corollary 9. Suppose $\prod R_{\alpha}$ is imbeddable in a zero-dimensional ring. If $P_{\alpha}$ is a height-zero prime of $R_{\alpha}$ for each $\alpha \in A$, then there exists a positive integer $n$ such that $\left\{\alpha \in A \mid \eta\left(\left(R_{\alpha}\right)_{P_{n}}\right)>n\right\}$ is finite.

We remark that another corollary to Theorem 7 is the known result that an arbitrary product $\Pi D_{\alpha}$ of integral domains $D_{\alpha}$ is imbeddable in a zerodimensional ring (for example, in $\prod K_{\alpha}$, where $K_{\alpha}$ is the quotient field of $D_{\alpha}$ ). This known result is, of course, also a consequence of the fact that an arbitrary reduced ring is imbeddable in a von Neumann regular ring [6, p. 9].

We have determined equivalent conditions for an arbitrary product $\prod R_{\alpha}$ to be imbeddable in a zero-dimensional ring. These conditions are stated in Theorem 10, but the criteria of that result are not very definitive in that they are difficult to apply in the general case. Because the proof of Theorem 10 requires no ideas that are essentially different from those already employed in this paper, we omit the proof. The statement of Theorem 10 uses some new notation. Suppose $S$ is a ring imbeddable in a zero-dimensional ring. Let $\mathscr{P}(S)$ be the set of all collections $\left\{Q_{\lambda}\right\}$ of primary ideals of $S$ with intersection (0). For $\left\{Q_{\lambda}\right\} \in \mathscr{P}(S)$ and for $a \in S$, define $\mu\left(a,\left\{Q_{\lambda}\right\}\right)$ to be

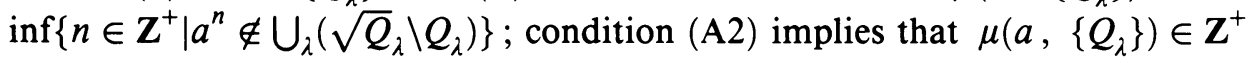
for some $\left\{Q_{\lambda}\right\} \in \mathscr{P}(S)$. Define $\mu\left(S,\left\{Q_{\lambda}\right\}\right)$ to be $\sup \left\{\mu\left(a,\left\{Q_{\lambda}\right\}\right) \mid a \in S\right\}$, and define $\mu(S)$ to be $\inf \left\{\mu\left(S,\left\{Q_{\lambda}\right\}\right) \mid\left\{Q_{\lambda}\right\} \in \mathscr{P}(S)\right\}$.

Theorem 10. Suppose $\left\{R_{\alpha}\right\}_{\alpha \in A}$ is a family of rings, each imbeddable in a zerodimensional ring. The following conditions are equivalent.

(1) $R=\prod R_{\alpha}$ is imbeddable in a zero-dimensional ring.

(2) There exists a positive integer $n$ such that $\left\{\alpha \in A \mid \mu\left(R_{\alpha}\right)>n\right\}$ is finite.

\section{REFERENCES}

1. M. Arapovic, Characterizations of the 0-dimensional rings, Glas. Mat. 18 (1983), 39-46.

2. _. The minimal 0-dimensional overrings of commutative rings, Glas. Mat. 18 (1983), 4752.

3. $\ldots$, On the imbedding of a commutative ring into a 0-dimensional ring, Glas. Mat. 18 (1983), 53-59.

4. R. Gilmer and W. Heinzer, Products of commutative rings and zero-dimensionality, in preparation.

5. J. Huckaba, On valuation rings that contain zero divisors, Proc. Amer. Math. Soc. 40 (1973), 9-19.

6. __ Commutative rings with zero divisors, Marcel Dekker, New York, 1988. 
7. P. Maroscia, Sur les anneux de dimension zero, Atti. Accad. Naz. Lincei Rend. Cl. Sci. Fis. Mat. Natur. 56 (1974), 451-459.

8. N. Popescu and C. Vraciu, Sur la structure des anneaux absolutement plats commutatifs, J. Algebra 40 (1976), 364-383.

9. D. Underwood, On some uniqueness questions in primary representation of ideals, J. Math. Kyoto Univ. 9 (1969), 69-94.

10. O. Zariski and P. Samuel, Commutative algebra, vol. I, Springer, Berlin, 1975.

Department of Mathematics, Florida State University, Tallahassee, Florida 32306

Department of Mathematics, Purdue University, West Lafayette, Indiana 47907 